\title{
Simulation and Optimization of Faceted Structure for Illumination
}

\author{
Lihong Liu ${ }^{\mathrm{a}, \mathrm{b}^{*}}$, Thierry Engel ${ }^{\mathrm{a}, \mathrm{b}}$, Manuel Flury ${ }^{\mathrm{a}, \mathrm{b}^{*}}$ \\ ${ }^{a}$ Laboratoire des Sciences de l'Ingénieur, de l'Informatique et de l'Imagerie (Icube), UDS-CNRS, \\ 300 Boulevard Sébastien Brant, CS 10413, F-67412 Illkirch Cedex, France \\ bnstitut National des Sciences Appliquées, 24 Boulevard de la Victoire, 67084 Strasbourg Cedex, \\ France
}

\begin{abstract}
The re-direction of incoherent light using a surface containing only facets with specific angular values is proposed. A new photometric approach is adopted since the size of each facet is large in comparison with the wavelength. A reflective configuration is employed to avoid the dispersion problems of materials. The irradiance distribution of the reflected beam is determined by the angular position of each facet. In order to obtain the specific irradiance distribution, the angular position of each facet is optimized using Zemax OpticStudio 15 software.

A detector is placed in the direction which is perpendicular to the reflected beam. According to the incoherent irradiance distribution on the detector, a merit function needs to be defined to pilot the optimization process. The two dimensional angular position of each facet is defined as a variable which is optimized within a specified varying range. Because the merit function needs to be updated, a macro program is carried out to update this function within Zemax. In order to reduce the complexity of the manual operation, an automatic optimization approach is established. Zemax is in charge of performing the optimization task and sending back the irradiance data to Matlab for further analysis.

Several simulation results are given for the verification of the optimization method. The simulation results are compared to those obtained with the LightTools software in order to verify our optimization method.

Keywords: Faceted Structure, incoherent light, illumination, optimization
\end{abstract}

*lihong.liu@ unistra.fr, manuel.flury@insa-strasbourg.fr; phone (+33) 368854632 or (+33) 388144747 ; icube-ipp.unistra.fr

\section{INTRODUCTION}

In the area of non-imaging optics, the redistribution of incoherent light for a specific target illumination is an important field of research and has a variety of applications. For example, the optical elements with specific reflection could be used for the material design in architecture and industry [1]. In addition, it can be applied in off-axis illumination to double the resolution for optical lithography systems [2,3]. Redirecting the incoherent light can be made through several methods. Freeform mirrors have been investigated with the decomposition of the reflective surface into a set of paraboloids [4]. However, a large amount of variables need to be optimized for the configuration of the integral mirror. Weyrich et al. proposed a method coming from computer graphics for the redirection of the Bidirectional Reflectance Distribution (BRDF) [1]. They use a series of analytical ideas to calculate the reflective faceted surface and using a spherical convolution with the Point Spread Function of a facet. Another solution is the case of Digital Mirror Devices developed by Texas Instruments [5,6].

The goal of the present work is the redirection of incoherent light. We propose to use the photometric approach which is different from popular methods coming from freeform optics or scalar electromagnetism. An original approach for shaping an incoherent beam is investigated. Our aim is to demonstrate the opportunity to make a redirection of the light using a surface containing only facets with a specific angular distribution. We are using only photometric levels and nonsequential ray-tracing of the light, without physical information (polarization, phase). The advantage of our approach is that it does not depend on the coherent properties of the light: it is just a redirection of the light using "small mirrors". The redistribution of the beam is accomplished by iterative optimization in the non-sequential mode of Zemax OpticStudio15 software which is normally used in computing assisted design in optics. Different specific examples with faceted surfaces will be given to show our optimization method in the paper. The facet model has also been transferred to LightTools software to see if there are some discrepancies. 


\section{OPTICAL CONFIGURATION}

The optical configuration we used is simply made of an incoherent source with collimated uniform irradiance distribution, a reflective faceted surface and a detector. As the quality of the target light distribution depends on the dispersion of the material in the case of transmission and as the color artifact will probably disturb the final results, we choose the reflective configuration at first. The material of the reflective surface is then assumed to be a perfect mirror. The reflective surface is composed by a set of square facets. Each facet is a square volume with equal size. As our target is the redistribution of the incoherent irradiance, the photometric approach is our mainly employing theory. Based on this theory, the size of each facet needs to be large enough compared to the wavelength. In this case, the size of each facet is defined to be $2 \mathrm{~mm}$. To reduce the complexity of the design process, the number of facets is set to be 6 by 6 .

\subsection{Initial configuration}

Before the optimization process, the tilt angle of each facet is zero. In the initial configuration, the overall reflective surface composing 6 by 6 facets is flat. The initial reflective configuration is depicted in figure 1 .

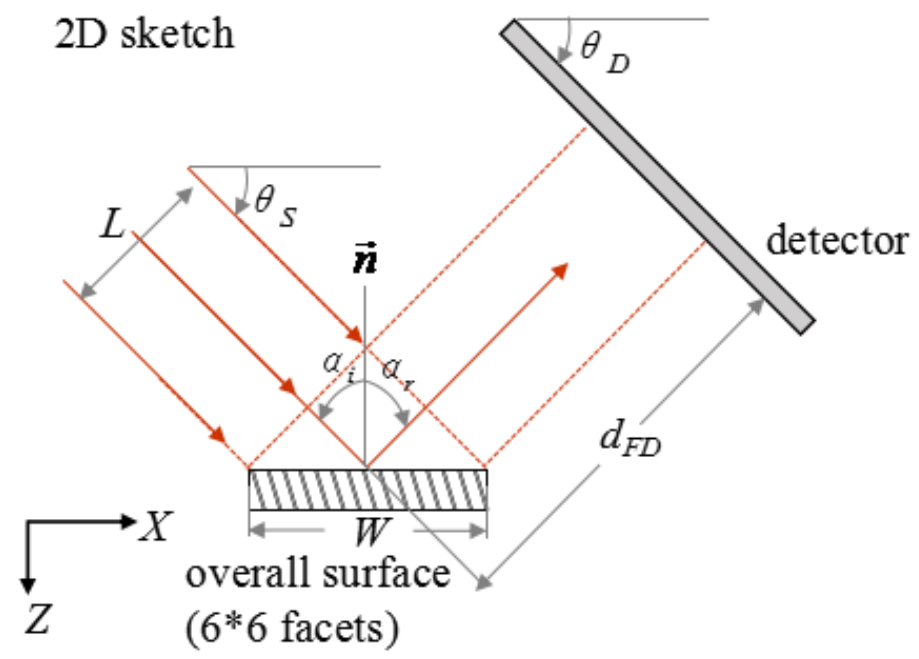

Figure 1: 2D sketch of initial configuration in the $X-Z$ plane. $W$ is the width of the overall surface. $i$ is the normal of the overall surface. $\alpha_{i}$ is the incident angle in the initial configuration. $\alpha_{r}$ is the correspondent reflected angle. $d_{F D}$ is the distance between the detector and the overall surface, it is parallel to the reflected beam. $L$ is the width of the incident beam. $\theta_{S}$ is the tilt angle between the axis $x$ and the parallel incident beam. $\theta_{D}$ is the tilt angle between the axis $x$ and the detector. The center of the reflective surface is noted as $O$, the coordinate of $O$ is $(0,0)$. The center of the source is noted as $S$, the coordinate of $S$ is $(-5,-5)$. The center of the detector is noted as $E$, the coordinate of $E$ is $(100,-100)$.

As shown in the above figure, the reflective surface is uniformly illuminated by the incident beam. The reflective surface containing 6 by 6 square facets is designated to realize the goal of redirecting the incoherent light into the desired distribution. For the feasibility of the following optimization process, the incident beam struck the initial reflective surface with 45 degrees, and the initial reflected beam struck the detector with 90 degrees. So the tilt angle of the incident beam $\theta_{S}$ is 45 degrees, and the tilt angle of the detector $\theta_{D}$ is also 45 degrees. We define the ratio between the distance $d_{F D}$ and the width of the overall surface $W$, which is proportional to the $f$-number of the system. In our case, this ratio should be larger than 10 to avoid the problem of convergence in the numerical optimization process:

$$
\frac{d_{F D}}{W}>10
$$

As the width of each facet is $2 \mathrm{~mm}$, the width of the overall surface is $12 \mathrm{~mm}$. As the slope of the line $O E$ is -1 , the distance $d_{F D}$ equals to the size of the segment $O E$. By calculating the distance between the center of the reflective surface and the center of the detector, the distance $d_{F D}$ equals to $141.42 \mathrm{~mm}$. So the value of the expression (1) is 11.8 . The shape of the incident beam is set to be square. The total power of the source is $1 \mathrm{~W}$. In the initial configuration, the irradiance distribution on the detector is shown in the following figure 


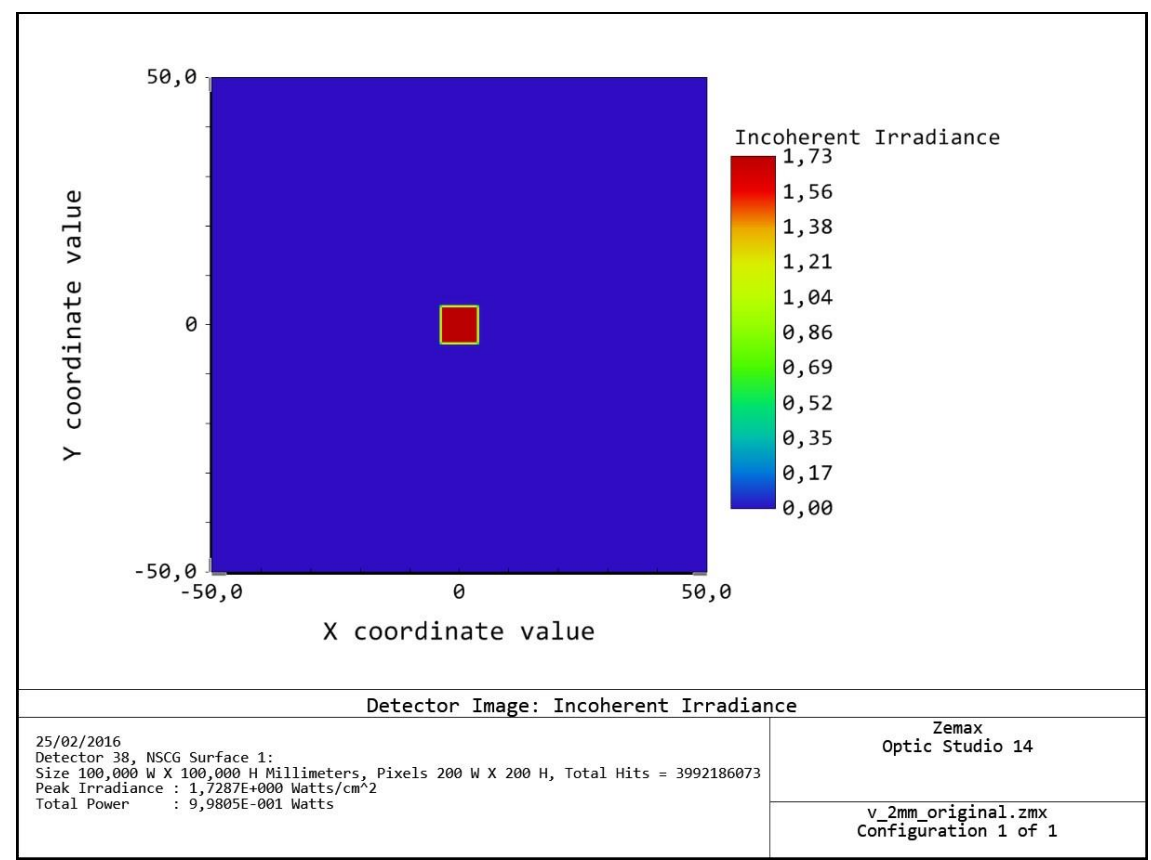

Figure 2: Irradiance distribution on the detector in the initial configuration

The irradiance spot on the detector is a square. The width of the spot is the equal size of the incident beam. The width of the detector is $100 \mathrm{~mm}$, and the number of pixels on the detector is $200 \times 200$.

\subsection{The configuration during the optimization process}

For our goal of redirecting the incoherent beam, the angular position of the front face of the facet needs to be optimized in two dimensions. As shown in figure 3, the tilt of the front face is determined by the tilt angle along the axis $X$ and the tilt angle along the axis $Y$ together. So the angular position is defined by the front $X$ angle and the front $Y$ angle. Thus, the optimization process should be carried out by iteratively changing the angular position in two dimensions, until the desired irradiance distribution is obtained on the detector. The 3D sketch containing 2 by 2 facets is shown in figure 3

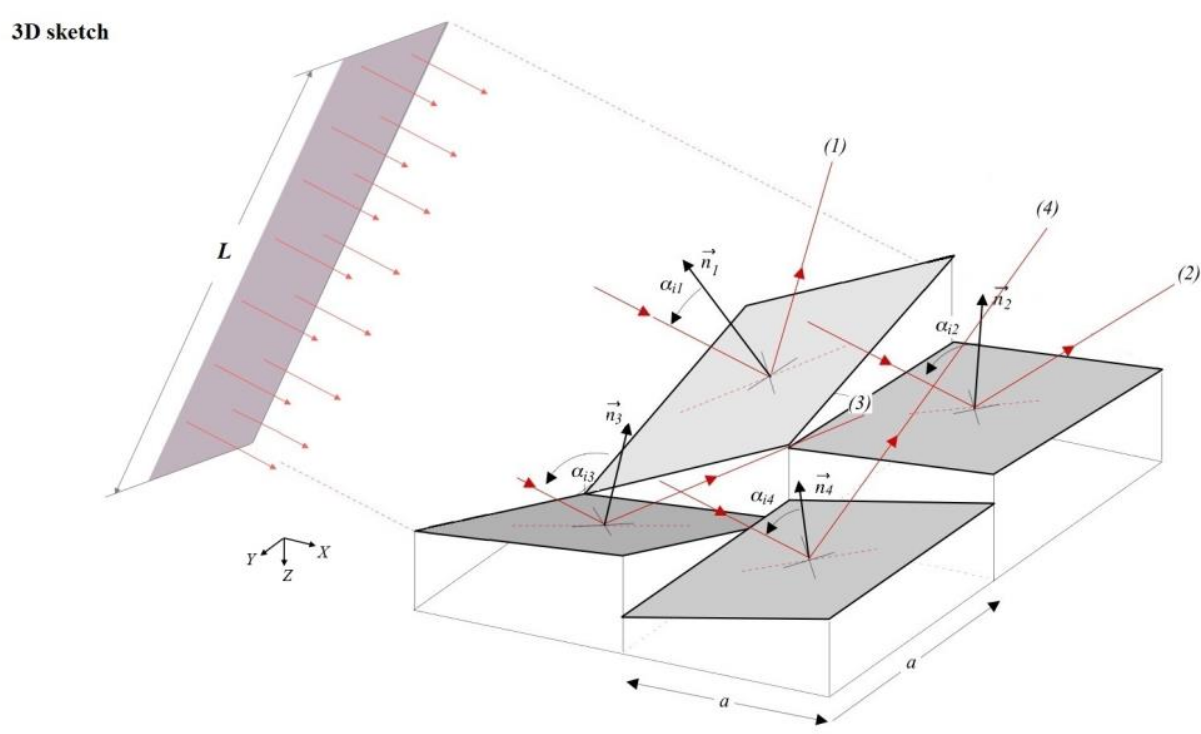

Figure 3: 3D sketch of the configuration used for the optimization process. $L$ is the width of the incident beam. $a$ is the size of the facet. After optimization, the normal vectors of each facet are changed from $\vec{n}$ to $\vec{n}_{1}, \vec{n}_{2}, \vec{n}_{3}$, $\vec{n}_{4}$. The correspondent incident angles are changed from 45 degrees to $\alpha_{i 1}, \alpha_{i 2}, \alpha_{i 3}, \alpha_{i 4}$. 
The reflective surface is composed by a number of facets, each facet is illuminated uniformly by a square sub-beam. In figure 3, the normal $\vec{n}$ is determined by the tilt angles of the front face along the axis $X$ and the axis $Y$. When the normal $\vec{n}$ changes with angle $\alpha$, the reflected sub-beam will be diverged with $2 \alpha$. Based on this property, the angular position of facets will be optimized iteratively, until the desired irradiance distribution is obtained on the detector.

\section{OPTIMIZATION PROCESS}

\subsection{Merit function}

As mentioned before, the optimization process is made of several loops. The merit function is defined to optimize the angular positions in every loop. As we know, the merit function in Zemax is composed by different operands. In our case, the operands are separated into three parts. The first part is the restriction on the angular range of each facet. If the angular position is too large, the ray will be reflected to exit the reflective system. To avoid the unnecessary loss of the optical energy, the restriction on the angular range should be added in the merit function. In our case, each facet is a square volume with the equal size of $2 \mathrm{~mm}$. To maintain a regular geometrical shape of the facet, so the angular range is $(-30,30)$ in each dimension. The first part just acts on limiting the varying range of the angular positions, it has no impact on the result of the merit function. So the weight of operand in the first part is zero. The second part of the merit function is composed by two operands for getting the irradiance data from each pixel on the current detector. As our optimization is processed in the software of Zemax OpticStudio 15 , the first operand NSDD $(0,0,0,0)$ is used to erase the current detector, the second operand NSTR $(0,0,0,0,0)$ is used to perform the non-sequential ray-tracing in Zemax. For the ray-tracing, the number of rays is set to be 5000. In other words, the second part contains two operations. One is for clearing the detector, the other one is for the non-sequential ray-tracing. The second part is just used for performing the essential operations, so the second part has no impact on the result of the merit function. Thus, the weight of operands in the second part is also zero. After the operation of the second part, the third part of operands are used to obtain the irradiance data from each pixel. As the number of pixels on the detector is set to be $200 \times 200,40000$ operands of NSDD $(38, k, 1,0)(k=1 \cdots 4000)$ are used. 38 defines the object number of detector, 1 defines the unit for the returned value is flux/area.

We can conclude that the operands of the first part impose the restrictions on the varying range of angles. The second part clears the detector and performs the non-sequential ray-tracing. When the optimization begins to run, merit function is updated firstly. In the non-sequential mode, the operands of the second part are essential for updating the merit function. Then the third part of operands obtain the current irradiance data from each pixel. As the weights for the first and the second part are zero. So the merit function could be written in the following form

$$
M F^{2}=\frac{\sum_{k_{1} \in \text { target }} m_{k_{1}}\left(R_{k_{1}}-T_{k_{1}}\right)^{2}+\sum_{k_{2} \in \text { non }- \text { target }} m_{k_{2}}\left(R_{k_{2}}-T_{k_{2}}\right)^{2}}{\sum_{k_{1} \in \text { targ } e t} m_{k_{1}}+\sum_{k_{2} \in \text { non }- \text { arget }} m_{k_{2}}}
$$

In equation (2), $M F$ is the result of the merit function, $R$ is the obtained irradiance data from each pixel, $T$ is the objective irradiance value for each pixel, $m$ is the weight. The pixels on the detector are separated into two areas. One area is composed by the pixels $k_{l}$ which are within the target area. The other area is composed by the pixels $k_{2}$ within the nontarget area. The value of the weight $m$ is defined to be 1 . So the merit function could be simplified as

$$
M F^{2}=\frac{\sum_{k_{1} \in \text { target }}\left(R_{k_{1}}-T_{k_{1}}\right)^{2}+\sum_{k_{2} \in \text { non-target }}\left(R_{k_{2}}-T_{k_{2}}\right)^{2}}{40000}
$$

In this merit function, when the actual irradiance data $R$ approaches the objective value $T$, the result of the merit function decreases. So we can conclude that when the value of the merit function decreases to 0, the desired irradiance distribution will be achieved. But it is not necessary to reach this ideal condition. As long as the merit function is decreased to be a relatively low value, a precise irradiance distribution could be obtained on the detector which will be verified by the optimization results in the fourth section. 


\subsection{Manual optimization}

After defining the merit function, the optimization process in the manual mode is designed. The optimization process needs to be accomplished by several loops. In every loop, the 2D angular positions are variables, and the Orthogonal Descent algorithm is used to decrease the value of the merit function. When the algorithm gets the local minimum, one optimization loop is ended. Then the operations of clearing the detector and non-sequential ray-tracing are executed manually, the current irradiance distribution on the detector will be compared with the target. If it complies with the target, the optimization will be terminated. If not, the merit function will be refreshed by executing a ZPL macro, then the next loop of optimization will continue to be run.

Zemax Programming Language (ZPL) is a macro language that allows the customer to create its own functions. For the several of operations in Zemax, correspondent commands have been retained in ZPL. The ZPL macro can be written in the text file and saved in .ZPL format. By executing the .ZPL file, user defined operations can be performed in Zemax. In our case, the merit function needs to be updated by using the ZPL macro. As explained previously, in the non-target area, the positions of the pixels with the non-zero irradiance data have changed after one loop of optimization. So the purpose of the ZPL file is to refresh the positions of the pixels in the non-target area.

In the macro program, the two commands for clearing the detector and the non-sequential ray-tracing are applied firstly. Then the irradiance data from each pixel are obtained by using the numeric function of NSDD, which is also used as the operand in the merit function. In the non-target area, the positions of pixels with the non-zero irradiance value are extracted in macro program. The positions are rewritten into the NSDD operands in the merit function. Thus the correspondent operands $\operatorname{NSDD}\left(38, k_{2}, 1,0\right)$ are refreshed in the merit function. Every time after one optimization loop is ended, the ZPL file will be executed to refresh the data $k_{2}$ in the function $\sum_{k_{2} \in \text { non-target }}\left(R_{k_{2}}-T_{k_{2}}\right)^{2}$. Then the next loop of optimization continues to be run, until the desired irradiance distribution is achieved on the detector. The process is demonstrated in the figure 4

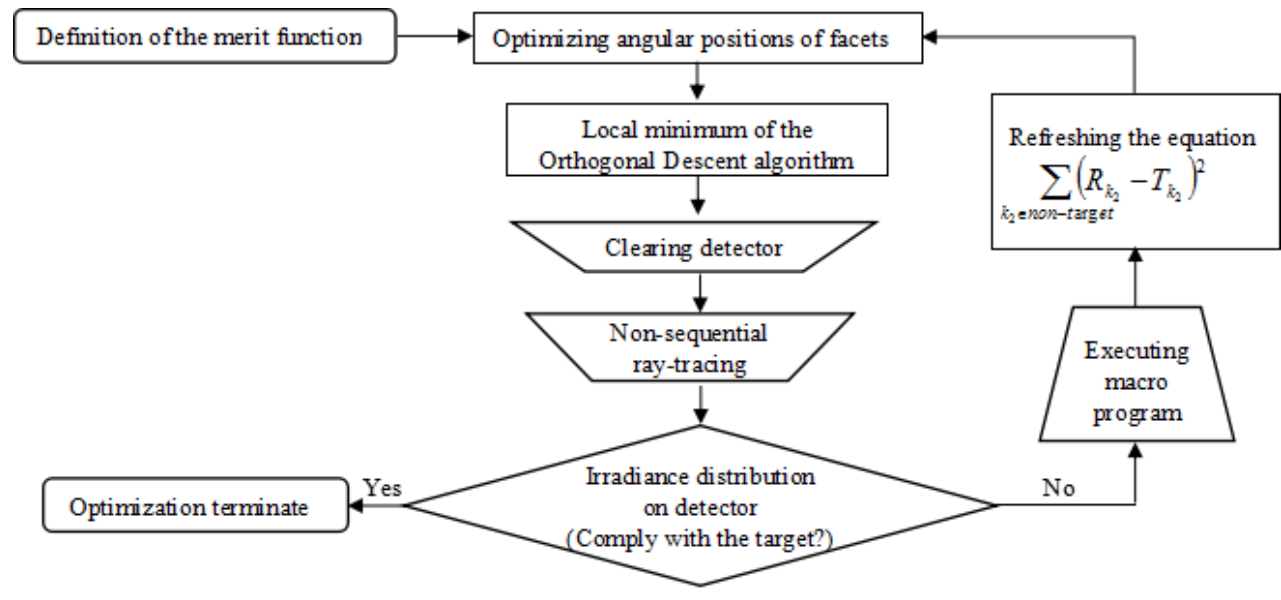

Figure 4: Manual optimization process using Zemax OpticStudio 15.

As shown in the figure 4, the optimization process is mainly composed by the definition of the merit function, the definition of the macro program and the optimization. Several cycles of the optimization are performed until the irradiance distribution complies with the requirement. In the flow chart, almost every step of the operation needs to be executed manually, it is very time consuming.

\subsection{Automatic optimization}

As described in the last section, each part of the optimization process needs to be manipulated manually. An automatic optimization is established to simplify the process. A macro program which is written in the ZPL file is used to realize the automatic function as shown in figure 5. 


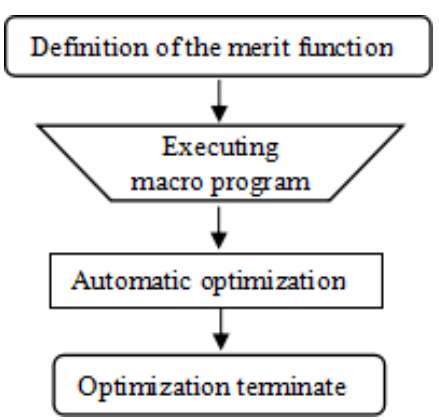

Figure 5: Automatic optimization process using Zemax OpticStudio 15.

Compared with the figure 4 , the automatic macro program comprises the previous manual operations of updating the merit function, optimization and the calculation of the irradiance difference. Each time one optimization loop is ended, the value of the irradiance difference is calculated. If the value is bigger than the pre-set standard value, another optimization loop is processed. When the value gets below the standard one, the optimization is terminated. Within Zemax software, all these operations can be realized by directly executing the corresponding macro functions in the ZPL file. Thus, the macro program which contains the corresponding macro functions and the required calculations can replace the previous manual optimization process. By directly running the macro program, the angular position will be optimized automatically and the irradiance distribution will be achieved. To simplify the complexity of the calculation of the irradiance difference, a simple function is used as following

$$
S F=\sum_{k_{2} \in \text { non-t } \arg e t} R_{k_{2}}
$$

In equation (4), $k_{2}$ is the position of the pixel with the non-zero irradiance value in the non-target area, $R$ is the corresponding irradiance value, the result of the $S F$ is the sum of all the non-zero irradiance data within the non-target area. When the value of $S F$ is smaller than the preset standard value, the desired irradiance distribution can be achieved on the detector.

\section{RESULTS}

\subsection{Results with different irradiance distributions}

Two categories of results have been obtained for the verification of the optimization theory. The processor of the computer is an Intel Xeon E3-1270 3.50 GHz. The type of the operating system is with 64 bits, 4 cores and 32Go RAM. Firstly, the target on the detector is two horizontal spaced spots, symmetrical with the vertical axis $X=0$. The source is monochromatic, the wavelength is $550 \mathrm{~nm}$. The optimization results are shown in figure 6

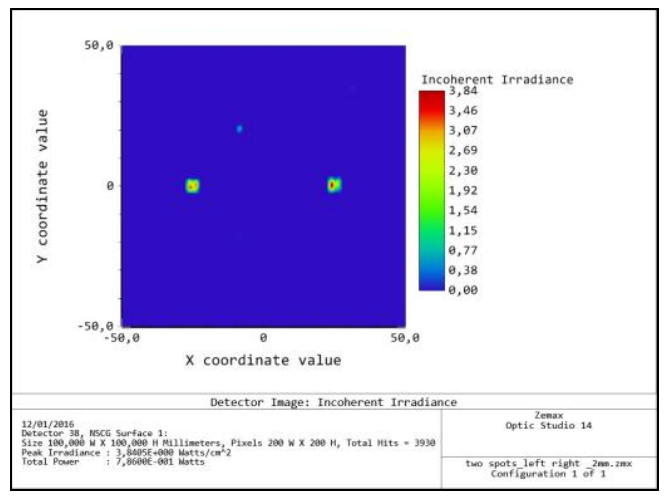

(a) Irradiance distribution of two horizontal spaced spots.

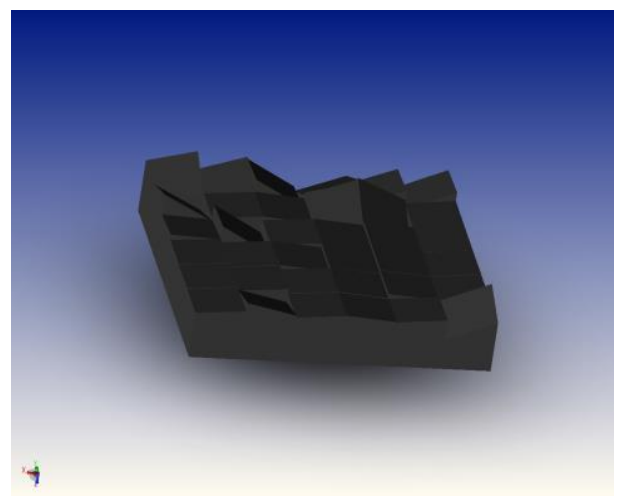

(b) Optimized structure using a 6x6 facets sample.

Figure 6: Irradiance distribution at one wavelength $(550 \mathrm{~nm})$ and facet structure after optimization. 
The first result is obtained by using the optimization process in the automatic mode. After 3 loops of optimization, the results have been obtained. The time of the optimization is 1.44 hours. As we can see in figure 6(a), the $y$ coordinate for the center of the two spots is $0.25 \mathrm{~mm}$, the $x$ coordinate for center of the left spot is $-25.25 \mathrm{~mm}$, and the $x$ coordinate for the center of the right spot is $25.25 \mathrm{~mm}$.

Secondly, the target on the detector is two vertical spaced spots, symmetrical with the horizontal axis $Y=0$ (see figure 7).

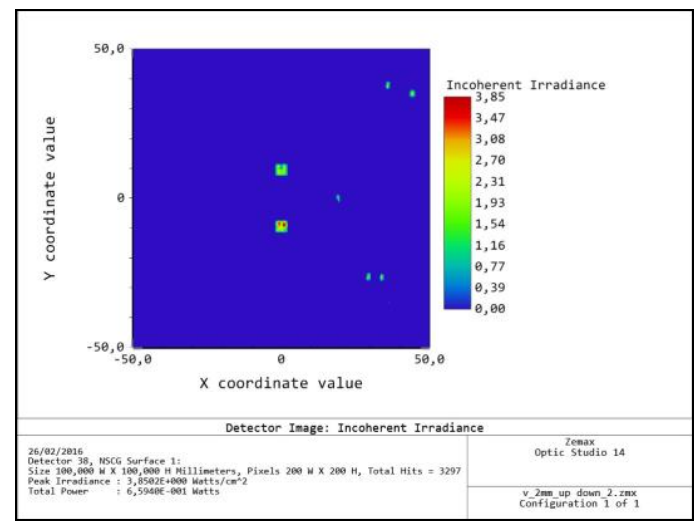

(a) Irradiance distribution of two vertical spaced spots.

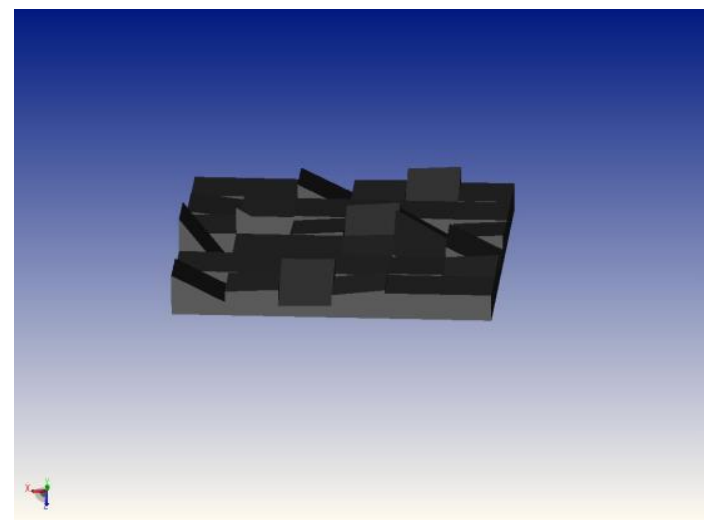

(b) Optimized structure using a 6x6 facets sample.

Figure 7: Irradiance distribution at one wavelength $(550 \mathrm{~nm})$ and facet structure after optimization.

This result is obtained manually. It takes about 1.5 hours to get the result. The number of loops is 3 . As we can see in the figure 7(a), the $x$ coordinate for the center of the two spots is zero. The $y$ coordinate for the center of the upper spot is $9.25 \mathrm{~mm}$, the $y$ coordinate for the center of the lower spot is $-9.25 \mathrm{~mm}$. The coordinate of the detector is changed to be $(200,-200)$ in the $x-z$ plane.

Moreover, the combination of different wavelengths has been tested as well. The influence of different wavelengths in the optimized structure is analyzed. We use a polychromatic source containing three wavelengths, red $(611 \mathrm{~nm})$, green $(549 \mathrm{~nm})$ and blue light $(464 \mathrm{~nm})$. The true color distribution is shown for the two horizontal spaced spots

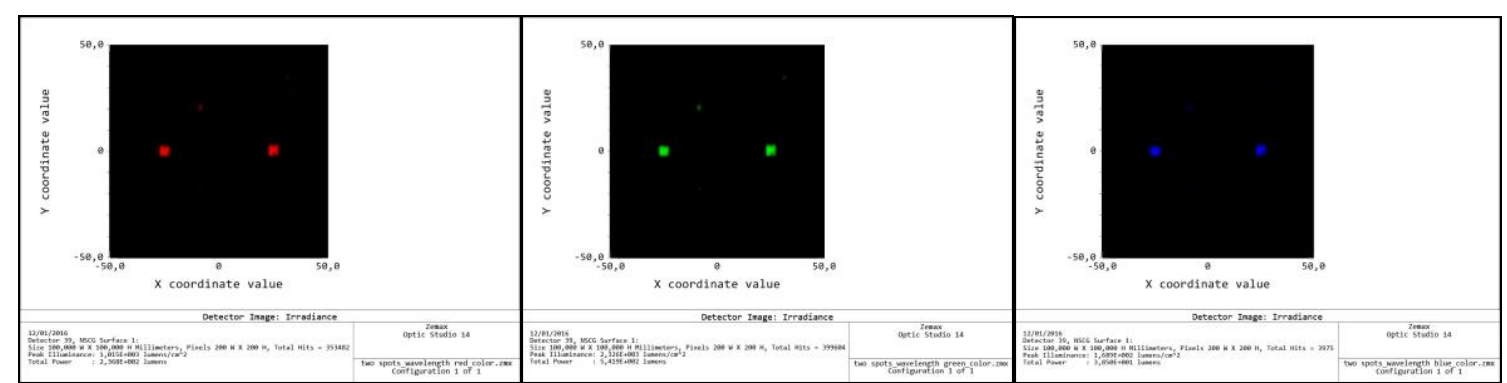

(a) Red $(611 \mathrm{~nm})$

(c) Blue $(464 \mathrm{~nm})$ (b) Green $(549 \mathrm{~nm})$

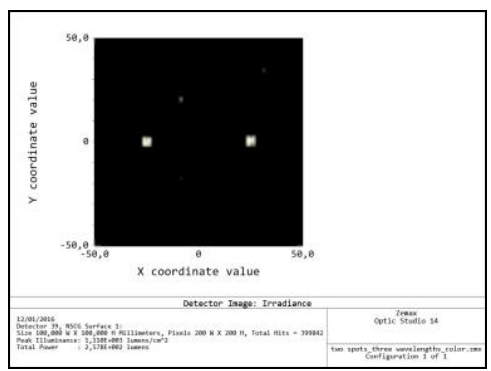

(d) 3 wavelengths combined with the same weight

Figure 8: True color distribution at different wavelengths. 
In the figure 8 , no influence of the wavelength is observed in the simulated image. The spots stay at the same place at any wavelength. This can be simply explained by the fact that we use perfect mirrors without diffusion or rough surfaces.

\subsection{Results comparison with LightTools}

The simulation results were compared to those obtained with the LightTools software in order to verify the image obtained in the detector plane placed at the right distance. The facet model is transferred from Zemax after optimization into LightTools software. The parameters of the optical system in LightTools are exactly the same as in Zemax. The source is polychromatic with three wavelengths, red $(611 \mathrm{~nm})$, green $(549 \mathrm{~nm})$ and blue light $(464 \mathrm{~nm})$. The irradiance distribution for the polychromatic source is shown in the following figure 9.

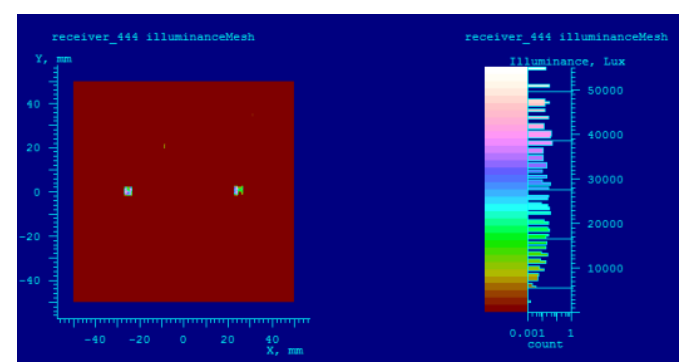

Figure 9: Simulation in LightTools for facet reflection at three wavelengths combined.

We can see in figure 9 that the position of the spots does not change comparing with Zemax software. There is also no color artifact. The simulation result in LightTools has further proved the optimization method for the redistribution of the incoherent light. We see also in the right histogram visible in the figure 9 that the spots are not exactly homogeneous: the optimization process was not taking account this criterion for the moment.

\subsection{Results calculation with Matlab}

Numerical measurements need to be made to assess the quality of the redistribution of the incoherent beam. The correlation between the desired irradiance distribution and the achieved one could be used as a metric or a quality factor. The correlation coefficient is calculated based upon the referenced functions in diffractive optics $[7,8]$

$$
C=\frac{\sum_{n=1}^{N} \sum_{m=1}^{M} \frac{O_{n, m} \cdot U_{n, m}}{N \cdot M}-\left[\sum_{n=1}^{N} \sum_{m=1}^{M} \frac{O_{n, m}}{N \cdot M}\right] \cdot \sum_{n=1}^{N} \sum_{m=1}^{M} \frac{U_{n, m}}{N \cdot M}}{\sqrt{\left[\sum_{n=1}^{N} \sum_{m=1}^{M} \frac{O_{n, m}^{2}}{N \cdot M}-\left[\sum_{n=1}^{N} \sum_{m=1}^{M} \frac{O_{n, m}}{N \cdot M}\right]^{2}\right] \cdot\left[\sum_{n=1}^{N} \sum_{m=1}^{M} \frac{U_{n, m}^{2}}{N \cdot M}-\left[\sum_{n=1}^{N} \sum_{m=1}^{M} \frac{U_{n, m}}{N \cdot M}\right]^{2}\right]}}
$$

$O_{n, m}$ is the desired irradiance value for each pixel. $U_{n, m}$ is the numerically reconstructed wavefront complex amplitude sampled at location referred $(n, m)$, in our case $U_{n, m}$ is the obtained irradiance value from each pixel. $N$ and $M$ are the number of pixels in each dimension, so $N=M=200$.

Based on the MZDDE toolbox, the dynamic data transfer could be realized between Zemax and Matlab. Matlab sends out a series of commands to Zemax. Zemax performs the correspondent operations and transfers the required data back to Matlab. Firstly the link between Zemax and Matlab is initialized. After the link is initialized successfully, Matlab sends the commands of clearing the detector and non-sequential ray-tracing to Zemax, then Zemax is asked to transfer all the irradiance data back to Matlab. Thus the correlation coefficient can be calculated in Matlab. $O_{n, m}$ and $U_{n, m}$ are normalized before calculation. And the value of the correlation coefficient $C$ is always in the interval $[0,1]$.

Table 1. Correlation coefficient for the irradiance distribution of two horizontal spaced spots at red, green, blue and three wavelengths combined. These values were calculated with Zemax.

\begin{tabular}{|c|c|c|c|c|}
\hline Item & Red & Green & Blue & Three wavelengths \\
\hline Correlation & 0.8993 & 0.8999 & 0.8996 & 0.8965 \\
\hline
\end{tabular}


According to the calculation result of the correlation coefficient in table 1 , the achieved irradiance distribution approaches to the target with a small deviation. These results have further proved the feasibility of the optimization method for the redistribution of the incoherent beam.

\section{CONCLUSION AND OUTLOOK}

A reflective optical system has been set up for the redirection of the incoherent beam. The optical configuration is simply composed by a source, a reflective surface and a detector. The reflective surface is made of $6 \times 6$ square facets, the dimension of the facets is $2 \mathrm{~mm}$. The redistribution of the irradiance distribution is realized by the optimization of the angular positions of the facets. For achieving different irradiance distribution, merit function is defined to control the optimization process. Based on the merit function, manual optimization is established in the first step. Then automatic optimization is set up later to simplify the manual optimization process. By directly running the macro program, the desired irradiance distribution could be achieved automatically. Several optimization results have been got for the verification of the optimization method. Monochromatic irradiance distributions of two spots with left-right position and up-down position are obtained. Polychromatic irradiance distributions for two spots with left-right position are also given for the demonstration of the color effect on the optimized structure. The correlation coefficient between the target and the achieved irradiance distribution is calculated for the confirmation of the optimization results.

The first results of the project are encouraging for the redirection of the polychromatic light without color artifacts. Future work will involve using more complex targets, studying efficiencies and the influence of various parameters.

\section{ACKNOWLEDGEMENTS}

The authors would like to acknowledge the cooperation program with INSA Strasbourg and the China Scholarship Council for the PhD Grant of Lihong Liu. We thank Yoshitate Takakura from TRIO Group in ICube Laboratory for helpful discussions about optical simulations.

\section{REFERENCES}

[1] Weyrich, T., Peers, P., Matusik, W. and Rusinkiewicz, S., "Fabricating Microgeometry for custom surface reflectance," Proc. SIGGRAPH 28, 321-326 (2009).

[2] Wu, R., Li, H., Zheng, Z. and Liu, X., "Freeform lens array for off-axis illumination in an optical lithography system," Appl. Opt. 50(5), 725-732(2011).

[3] Wu, R., Liu, P., Zhang, Y., Zheng, Z., Li, H. and Liu, X., "A mathematical model of the single freeform surface design for collimated beam shaping," Opt. Express 21(18), 20974-20989 (2013).

[4] Michaelis, D., Kudaev, S., Steinkopf, R., Gebhardt, A., Schreiber, P. and Bräuer, A., "Incoherent beam shaping with freeform mirror," Proc. SPIE 7059, 705905 (2008).

[5] Liang, J., Kohn Jr, R.N., Becker, M.F. and Heinzen, D.J., "High-precision beam shaper for coherent and incoherent light using DLP spatial light modulator," Proc. SPIE 7932, 793208 (2011).

[6] Liang, J., Kohn Jr, R.N., Becker, M.F. and Heinzen, D.J., "Evaluation of DMD-based high-precision beam shaper using sinusoidal-flattop beam profile generation," Proc. SPIE 8130, 81300C (2011).

[7] Jennison, B.K., Allebach, J.P. and Sweeney, D.W., "Iterative approaches to computer-generated holography," Opt. Eng. 28(6), 286629 (1989).

[8] Bokor, N. and Papp, Z., “Monte Carlo method in computer holography,” Opt. Eng. 36(4), 1014-1020 (1997). 